\title{
Outrun radiation damage with electrons?
}

\author{
Ray F Egerton
}

\begin{abstract}
The diffract-before-destroy method, using 50- to 100-fs $x$-ray pulses from a free-electron laser, was designed to determine the three-dimensional structure of biological macromolecules in close to their natural state. Here we explore the possibility of using short electron pulses for the same purpose and the related question of whether radiation damage can be outrun with electrons. Major problems include Coulomb repulsion within the incident beam and the need for high lateral coherence, difficulties that are discussed in terms of existing and future electron sources. Using longer pulses of electrons appears to make the attainment of near-atomic resolution more feasible, at least for nanocrystalline particles, whereas obtaining this information from single-molecule particles in an aqueous environment seems a more distant goal. We also consider the possibility of serial crystallography using a liquid jet injector with a continuous electron beam in a transmission electron microscope (TEM).
\end{abstract}

Keywords: Macromolecules; Diffractive imaging; Cryomicroscopy; Radiation damage

\section{Background}

The understanding of biological processes at a cellular level requires a knowledge of the structure of macromolecules (such as proteins) or other entities (such as viruses). Ideally, a resolution better than $0.3 \mathrm{~nm}$ is desired and is achievable by means of $\mathrm{x}$-ray diffraction from a crystal, combined with algorithms to extract the phase of the diffracted beams to generate a three-dimensional structure, the so-called diffractive imaging [1]. Spreading the $\mathrm{x}$-ray dose over many identical units, as in a crystal, greatly reduces the problem of destruction of the molecules by radiation damage [2]. In some cases, however, crystals of sufficient size are difficult or impossible to grow [3] and prolonged x-ray diffraction from smaller crystals reintroduces the problem of damage.

An alternative method is cryo-TEM. Since electrons interact more strongly with solids, smaller crystals can be used, embedded in ice, and examined at low temperature $[2,4]$. Because electron lenses allow subatomic resolution, direct imaging can be used. Problems of specimen distortion under the beam are mitigated by the use of a fastreadout direct-exposure detector [5], allowing images to be read out rapidly and shifted into register [6]. Phase contrast is obtained by substantial defocus of the

Correspondence: regerton@ualberta.ca

Physics Department, University of Alberta, 116 St. and 85 Ave., Edmonton T6G 2E1, Canada specimen, although improved phase plates may achieve a better contrast-transfer function with an in-focus specimen [7].

One objection to the use of crystals (and cryogenic techniques) is that the outer (tertiary) structure of a biomolecule may differ from that in its in vivo cellular environment. The so-called single-particle imaging uses individual particles deposited in different orientations on a substrate, embedded in vitreous ice, or negatively stained. Two-dimensional images of thousands of particles are recorded at low electron dose (resulting in a poor signal/noise ratio) and are then used to construct the three-dimensional structure with subnanometer resolution, in a way similar to electron tomographic imaging [8].

\section{Methods}

\section{Diffract-before-destroy with $\mathrm{x}$-rays}

In response to the problems of radiation damage and the challenge of growing large crystals, a serial crystallography method was developed that takes advantage of the short (50 to $100 \mathrm{fs}$ ) but very intense pulses produced by an $\mathrm{x}$-ray free-electron laser (XFEL) [9]. The x-ray beam has typically been $2 \mu \mathrm{m}$ in diameter but will eventually be focused to $100 \mathrm{~nm}$ [9]. It is aimed at particles in the form of small crystals, single macromolecules, or viruses, contained in a liquid jet up to $2 \mu \mathrm{m}$ in diameter and traveling at about $10 \mathrm{~m} / \mathrm{s}$ or contained within aerosol droplets. Each particle is destroyed by the $\mathrm{x}$-ray pulse, but there are sufficient

\section{Springer}


$\left(\approx 10^{12}\right)$ photons within the first 50 fs to provide a diffraction pattern, from which the particle orientation can be determined. During this short time, the atomic structure of the particle remains intact and the measured structure is approximately that of the undamaged molecule. Repeating this process with some thousands or millions of particles provides enough information for the three-dimensional structure of the molecule to be determined by diffractive imaging. Because the phase of each diffracted beam must be well defined, a highly parallel $\mathrm{x}$-ray beam is required: the transverse coherence length should be at least twice the particle diameter [10].

In the limit of a very short pulse, wavelength-limited resolution would be possible for an arbitrarily small sample without damage, breaking the usual connection between dose, damage, and resolution [11]. For longer pulse durations, high-order diffraction spots are found to fade, as later portions of the pulse encounter previously damaged material, and resolution is lost.

The first XFEL experiments were conducted with 2-keV photons [12], the energy being subsequently increased to over $8 \mathrm{keV}$. The absence of damage was established using inorganic specimens and the technique then applied to biomolecular nanocrystals, where the coherent amplification of intensity due to Bragg scattering has allowed atomic resolution to be achieved in three dimensions [9]. Twodimensional protein crystals have also been examined [13]. For single particles such as a virus [14], the highest resolution achieved so far is about $12 \mathrm{~nm}$ for a single-shot (2-D) reconstruction, three-dimensional merging of singleparticle data having proved difficult in practice [14]. The interaction of the $\mathrm{x}$-ray pulses with the specimen has been modeled by computer calculations $[15,16]$, which were found to be consistent with experimental data [17].

\section{Advantages of electrons}

High-intensity $\mathrm{x}$-ray beams involve large and expensive facilities: there are less than 70 synchrotron sources and only six free electron lasers in the world at the present time. By comparison, instruments that use beams of high-energy (10 keV to $10 \mathrm{MeV}$ ) electrons are relatively compact and inexpensive. The transmission electron microscope (TEM) uses electromagnetic lenses to efficiently focus electrons and can provide direct images with atomic $(<0.2 \mathrm{~nm})$ resolution. Besides image formation, electron diffraction, electron energy loss spectroscopy (EELS), and $\mathrm{x}$-ray emission spectroscopy are routinely carried out in the TEM, adding to its analytical power. Admittedly, comparable analytical facilities could be added to $\mathrm{x}$-ray beam lines, as in the case of energydispersive spectroscopy carried out simultaneously with $\mathrm{x}$-ray diffraction [18].

Electron sources that deliver short $(<500 \mathrm{fs})$ pulses containing over $10^{6}$ electrons have been developed and used for single-shot electron diffraction measurements on inorganic specimens at $100 \mathrm{keV}$ [19-21] and $\mathrm{MeV}$ energies $[22,23]$. Therefore, the technology is well developed, and its development is continuing.

Electrons are scattered by solids much more strongly than $\mathrm{x}$-rays, so transmission measurements require a thin $(<1 \mu \mathrm{m})$ specimen. The fact that an electron beam requires high vacuum makes it harder to control the specimen environment, although lithography techniques have recently made environmental cells more practical. In addition, the liquid jet injector developed for XFEL applications has been successfully tested in a 200-kV TEM [24].

\section{Radiation damage}

Besides providing comparable analytical information, $\mathrm{x}$ ray and electron beams are also similar in their damaging effects. The main mechanism involved is ionization damage (radiolysis), in which valence or inner-shell electrons within the specimen are excited by inelastic scattering (of beam electrons) or absorption (of $\mathrm{x}$-rays). Although this primary process can itself result in the breakage of a chemical bond, most of the damage is believed to result from secondary electrons or photoelectrons that travel through the specimen and cause further bond breakage. For organic samples, various kinds of damage occur on different timescales [25], from electronic processes on the femtosecond scale, through homogeneous reaction on the nanosecond scale, to tertiary damage (e.g., protein unfolding) on the microsecond or a longer timescale.

Provided radiolysis is not outrun by using short pulses, the amount of damage is usually assumed to be proportional to the deposited energy, leading to its measurement in units of grays (= joules of deposited energy per kilogram). For $8-\mathrm{keV}(0.15 \mathrm{~nm}) \mathrm{x}$-rays, the damage per elastic scattering event is $10^{3}$ times larger than that for 80- to 500-keV electrons [26]. Because elastic scattering (diffraction) of electrons or $\mathrm{x}$-rays provides the information content of high-resolution structural images, electrons are capable of providing a substantially higher information/damage ratio.

\section{How electrons and $x$-rays differ}

The most significant difference between $x$-rays and electrons is that the latter carry an electrostatic charge, leading to Coulomb attraction or repulsion in the presence of other charged particles. So whereas the diffraction of $\mathrm{x}$-ray photons involves their interaction with atomic electrons, the elastic scattering (diffraction) of beam electrons arises from their Coulomb attraction towards atomic nuclei. The electronic charge also makes it easy to deflect and focus electrons with high efficiency, but their mutual repulsion in free space leads to spatial and energy broadening of an intense beam. 
A second difference between $x$-ray photons and electrons is that the latter carry more momentum for the same energy. Although the difference diminishes at higher energy, the $\mathrm{x}$-rays used for diffraction have photon energies of typically 1 to $10 \mathrm{keV}$, whereas the electrons that needed to penetrate even a thin $(<1 \mu \mathrm{m})$ specimen have kinetic energies of tens or hundreds of $\mathrm{keV}$. As a result, the electron/photon momentum ratio is typically 10 to 100 (see Figure 1).

One consequence of this higher momentum is that high-angle elastic collisions of electrons can create a second kind of radiation damage: knock-on or displacement damage, which is observed in conducting materials (e.g., metals) as the creation of defects or gradual amorphization of a crystalline specimen. But in most insulating materials, radiolysis provides a far more efficient damage mechanism; although knock-on damage must occur in organic materials, it is likely to be $10^{3}$ to $10^{6}$ times slower than radiolysis [27]. So in practice, the momentum associated with electrons does not appear to represent a significant additional problem with organic samples.

A third fundamental distinction is that electrons are fermions (whereas photons are bosons) and only one electron can occupy the same quantum state. This Pauli exclusion principle places a fundamental limit on the electron density, for a given energy range. Yet even for a field-emission electron source, the degeneracy factor amounts to only about $1.5 \times 10^{-5}$ at $300 \mathrm{keV}$ [28], meaning that the exclusion principle does not significantly affect present-day electron optics. In more practical terms, the highest current density currently achievable with an aberration-corrected lens is $J \approx 10^{7} \mathrm{~A} / \mathrm{cm}^{2}$, corresponding to an electron density $n=v^{-1}(J / e) \approx 0.5 \times 10^{16}$ electrons $/ \mathrm{cm}^{3}$. This value is small compared to the electron density in metals or the carrier concentration at

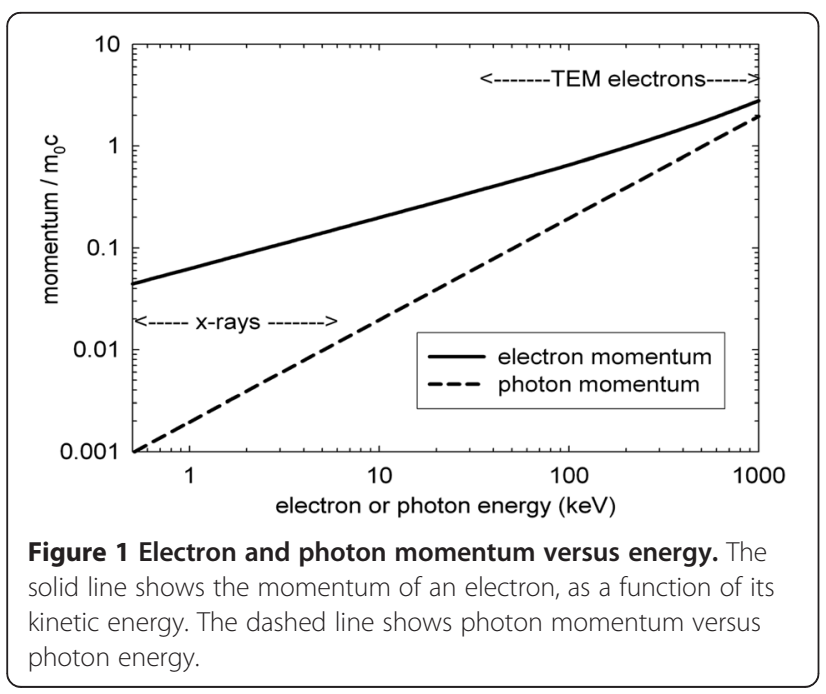

which degeneracy effects become important in semiconductors.

Experimentally, a further difference arises from the fact that electrons can lose varying amounts of energy in the specimen and continue to the detector, whereas $\mathrm{x}$ rays are annihilated when they create a photoelectron (which does not reach the detector). Consequently, there is a much higher inelastic background in the electron diffraction pattern, although this background can largely be removed by energy filtering.

\section{Results and discussion}

\section{Imaging with $\mathrm{x}$-rays and electrons}

X-rays can be focused by a diffraction grating or a zone plate to about $20 \mathrm{~nm}$, although with low efficiency, while electrons can be efficiently focused by an electromagnetic lens to subatomic dimensions. In the case of a beam-sensitive specimen, however, the usable resolution is limited by the radiation dose that the specimen can withstand before changing its structure. The doselimited resolution (DLR) of a two-dimensional image depends on the critical radiation dose $D_{\mathrm{c}}$ that creates significant damage and also on the image contrast and the efficiency with which the electrons or photons are used to form the image [29].

The DLR concept can be extended to threedimensional imaging, where radiation sensitivity sets a lower limit to the size $\delta$ of the cubic voxel that defines the resolution limit. Howells et al. [30] calculated the required dose for 3-D x-ray imaging with a signal/noise ratio of SNR $=5$ (the usual Rose criterion) and plotted it as a function of $\delta$, as in Figure 2. They also plotted the maximum tolerable dose $D_{\mathrm{c}}$ as a function of $\delta$, based on $\mathrm{x}$-ray and electron diffraction measurements. The point of intersection of these two curves defines the minimum useful voxel size $\delta$.

For comparison with the $\mathrm{x}$-ray case, we will calculate the required dose for electrons, making the same assumptions (which include large contrast between the chosen voxel and its surroundings). The number of electrons passing through a voxel is $D_{\mathrm{e}} \delta^{2}$, where $D_{\mathrm{e}}$ is the dose in electrons per unit area (as commonly used by electron microscopists). Neglecting dynamical effects, the fraction of electrons that are elastically scattered (diffracted) is $F=1-\exp \left(-\delta / \lambda_{\mathrm{e}}\right) \approx \delta / \lambda_{\mathrm{e}}$ for $\delta<<\lambda_{\mathrm{e}}$, where $\lambda_{\mathrm{e}}$ is the total mean free path for elastic scattering. The number of diffracted electrons is therefore $N_{\mathrm{e}}=D_{\mathrm{e}} \delta^{2} F \approx$ $D_{\mathrm{e}} \delta^{3} / \lambda_{\mathrm{e}}$. Regarding $N_{\mathrm{e}}$ as a signal, its associated shot noise is $N_{\mathrm{e}}^{1 / 2}$ and the signal/noise ratio is $\mathrm{SNR}=N_{\mathrm{e}} / N_{\mathrm{e}}^{1 / 2}$ $=N_{\mathrm{e}}^{1 / 2}$. The required electron dose is then $D_{\mathrm{e}}=N_{\mathrm{e}} /\left(\delta^{3} /\right.$ $\left.\lambda_{\mathrm{e}}\right)=(\mathrm{SNR})^{2}\left(\lambda_{\mathrm{e}} / \delta^{3}\right)$, where $\mathrm{SNR}=5$ is the required signal/noise ratio. For a direct comparison with $x$-rays, the radiation dose must be expressed in grays, using the relationship: 


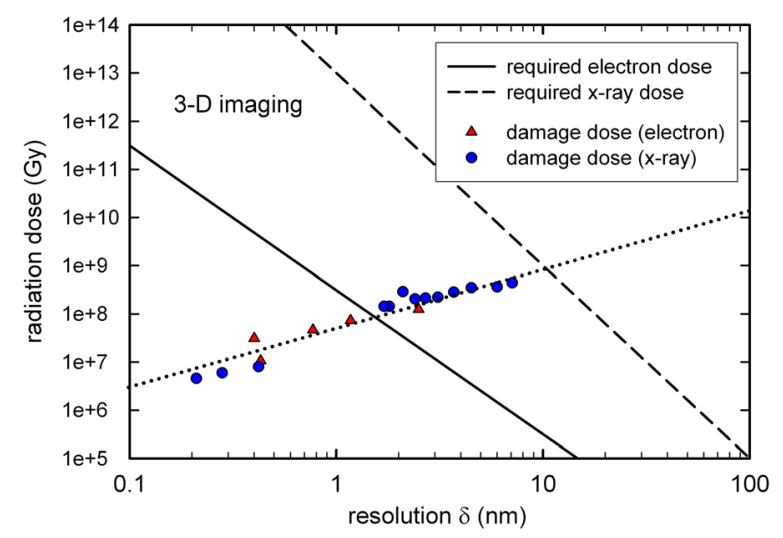

Figure 2 Required dose and tolerable dose for 3-D imaging using $\mathbf{x}$-rays and electrons. The dashed line is the $x$-ray dose required for three-dimensional imaging of a protein $\left(\rho=1.35 \mathrm{~g} / \mathrm{cm}^{3}\right)$ as a function of the voxel dimension $\delta$, according to [30]. The solid line is the required electron dose calculated from Equation 2, taking $\lambda_{\mathrm{e}} / \lambda_{\mathrm{i}}=3$ and $E_{\mathrm{m}}=35 \mathrm{eV}$. The dotted line represents the maximum tolerable dose, as determined by measurements by $x$-ray and electron diffraction measurements (round and triangular data points, respectively). For 3-D single-particle imaging, the best resolution corresponds to the intersection of the dotted line with the dashed line (for $x$-rays) or the solid line (for electrons).

$$
D(\mathrm{~Gy})=\left(D_{\mathrm{e}} / \rho\right)\left(E_{\mathrm{m}} / \lambda_{\mathrm{i}}\right)
$$

where $\rho$ is the specimen density (in $\mathrm{kg} / \mathrm{m}^{3}$ ), $E_{\mathrm{m}}$ is the mean energy loss (in J) per inelastic collision, and $\lambda_{\mathrm{i}}$ is the total mean free path (in $\mathrm{m}$ ) for inelastic scattering of the electrons. The required electron dose is then:

$$
\begin{aligned}
D(\mathrm{~Gy}) & =(1 / \rho)\left(E_{\mathrm{m}} / \lambda_{\mathrm{i}}\right)(\mathrm{SNR})^{2}\left(\lambda_{\mathrm{e}} / \delta^{3}\right) \\
& =(25 / \rho)\left(\lambda_{\mathrm{e}} / \lambda_{\mathrm{i}}\right)\left(E_{\mathrm{m}} / \delta^{3}\right)
\end{aligned}
$$

For an organic material, we can take $\rho=1.35 \times 10^{3} \mathrm{~kg} /$ $\mathrm{m}^{-3},\left(\lambda_{\mathrm{e}} / \lambda_{\mathrm{i}}\right) \approx 3$ (the value for carbon), and $E_{\mathrm{m}} \approx 35 \mathrm{eV}=$ $5.6 \times 10^{-18} \mathrm{~J}$, giving $D(\mathrm{~Gy})=(3.1 \mathrm{e} 8) / \delta(\mathrm{nm})^{3}$, independent of the electron energy (as is the damage dose, if expressed in grays). This $1 / \delta^{3}$ dependence is the same as that for $\mathrm{x}$-rays at small $\delta$ and is a property of incoherent imaging $[30,31]$.

The required electron dose given in Equation 2 is shown by the solid line in Figure 2. Its intersection with the damage dose line (dotted) defines a dose-limited resolution of about $1.5 \mathrm{~nm}$, a factor of ten better than the $\mathrm{x}$-ray value. Because of the $\delta^{3}$ dependence in Equation 2 , this result is in accord with a $10^{3}$ higher signal/ damage ratio for electrons [26].

Both $\mathrm{x}$-ray and electron calculations are optimistic in assuming negligible scattering outside the chosen voxel and an ideal detector $(\mathrm{DQE}=1)$ that collects all diffracted electrons. By using crystalline specimens, $x$-ray diffraction and cryoelectron microscopy overcome these limitations by combining information from many $(n)$ identical units, increasing the signal by a factor of $n$ or (equivalently) spreading the damage over $n$ molecules. The resolution is then improved by a factor $\approx n^{1 / 2}$, at the expense of placing the molecules in a less natural state.

Diffract-before-destroy provides an alternative solution: by outrunning the damage, the need for crystallization is (in principle) avoided. We now consider whether this option is feasible with electrons, which necessarily involves a pulsed electron beam.

\section{Properties of pulsed electron beams}

The radio-frequency photocathode source that injects electrons into a free-electron laser generates very short $(\approx 100 \mathrm{fs})$ pulses, containing at least $10^{6}$ electrons and representing an instantaneous current exceeding $1 \mathrm{~A}$ [32], so Coulomb repulsion is important. For the case of a continuous electron beam, Kruit and Jansen [33] discuss three aspects of this repulsion.

1. Global space charge: the effect of all other electrons on a given electron, causing a lateral broadening of the beam. This effect can be reduced by going to high (near-relativistic) beam energies, where the magnetic attraction of two parallel-moving electrons helps to compensate for their electrostatic repulsion. With ideal electron lenses, the space-charge effect can be compensated by refocusing [33].

2. Trajectory displacement [34], a stochastic (statistical) effect due to interaction between individual pairs of electrons, which cannot be removed by refocusing.

3. Energy broadening, also known as the Boersch effect, which destroys the longitudinal coherence of the beam and increases the chromatic aberration effects of any lenses that are used to focus it [35].

Kruit and Jansen give analytical formulas that help with understanding how these effects depend on parameters such as the electron path length, the beam divergence angle, and beam energy. Figure 3 a shows the predicted stochastic broadening of a $100-\mathrm{keV}$ continuous beam of diameter $200 \mu \mathrm{m}$, focused by an ideal lens towards a point over a distance of $10 \mathrm{~cm}$ (convergence semi-angle $=1 \mathrm{mrad}$ ). As the beam current $I$ is increased from $10 \mathrm{nA}$ to $1 \mathrm{~mA}$, the crossover diameter increases from subatomic dimensions to $10 \mu \mathrm{m}$ and the beam enters a regime where both the particle statistics and the current density profile are Gaussian. The beam diameter is then proportional to $I^{1 / 2}$, and increasing the current has no effect on the current density, which is limited to $J \approx 2,000 \mathrm{~A} / \mathrm{cm}^{2}$. If the beam energy is increased to $2.5 \mathrm{MeV}$, a similar behavior is predicted (Figure 3b) but the beam current can be increased to $I \approx 1$ A before the lateral broadening exceeds $1 \mu \mathrm{m}$, allowing a current density of $J \approx 60 \mathrm{MA} / \mathrm{cm}^{2}$. This example illustrates how 
a

Beam diameter for $V=100 \mathrm{kV}, \mathrm{L}=0.2 \mathrm{~m}, \alpha=1 \mathrm{mrad}, S_{c}=0.5$

Trajectory displacement: Kruit\&Jansen, Eqs.(7.68) - (7.70)

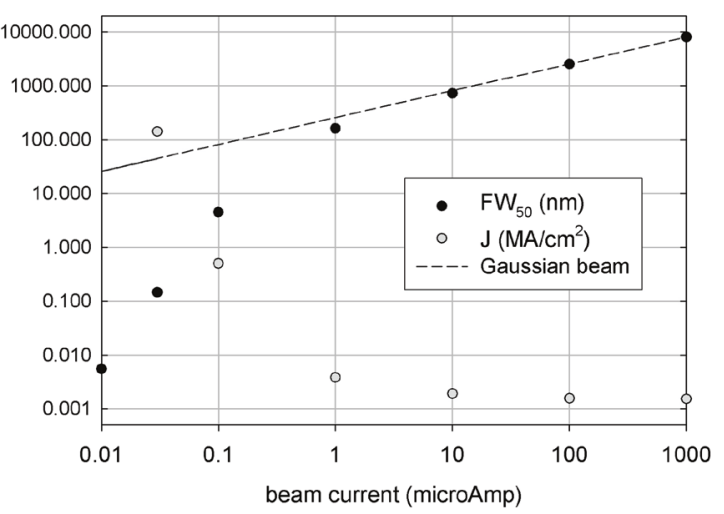

b

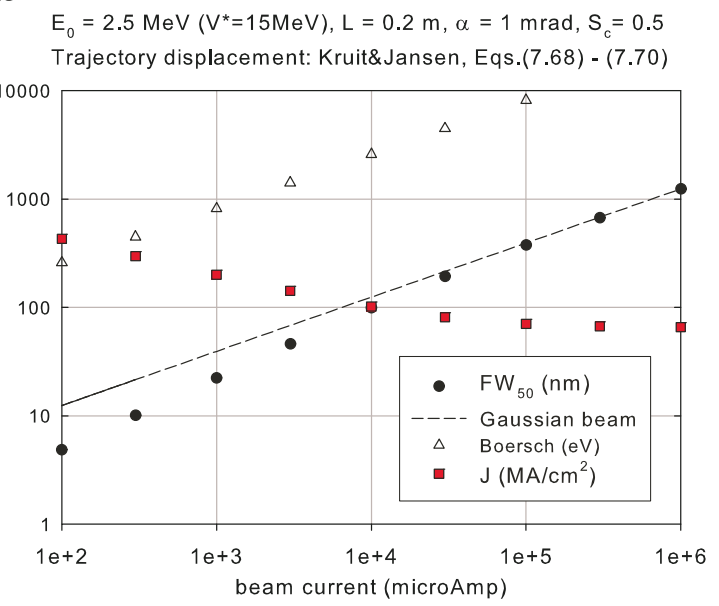

Figure 3 Stochastic broadening for $100-\mathrm{keV}$ and $2.5-\mathrm{MeV}$ electrons.

(a) The stochastic lateral broadening of a continuous electron beam (initial diameter $200 \mu \mathrm{m}$ ) focused towards a point over a distance of $10 \mathrm{~cm}$, calculated using the formulas of Kruit and Jansen [33] for an electron kinetic energy of $100 \mathrm{keV}$. (b) The corresponding quantities for 2.5-MeV electrons, together with the associated energy

broadening (triangles).

statistical broadening (as well as space-charge broadening) can be reduced by the use of a high beam energy [36].

Also shown in Figure $3 \mathrm{~b}$ is the predicted energy broadening of the beam, which increases proportional to $I^{1 / 2}$ and attains values $(>100 \mathrm{eV})$ that would make energy loss spectroscopy and focusing (for lenses with chromatic aberration) problematic.

For short pulses of electrons, stochastic broadening will be less. A minimum value (assuming broadening only close to the crossover and neglecting subsequent expansion) might be estimated by replacing the beam length $L$ by the electron bunch length $v T$, giving a reduction factor $(v T / \mathrm{L})^{2 / 3} \approx 300$. For annular dark-field ( $>5 \mathrm{mrad}$ ) imaging of 10 -ps pulses containing a million
5-MeV electrons, Armstrong et al. calculated the image blur profile to have a full width at half maximum (FWHM) width of $6.6 \mathrm{~nm}$ [37].

In practice, space-charge broadening appears to be the dominant effect in high-intensity beams. Figure 4 shows calculations of Renkai Li (personal communication) using General Particle Tracer software (3Dmesh, 3Dtreecode) to predict focusing of 100 -fs pulses (containing a million $2.5-\mathrm{MeV}$ electrons) by a magnetic field of peak strength $B_{0}$ and length $2.2 \mathrm{~cm}$ (FWHM), as in the objective lens of the 3-MeV UEM at Osaka University.

For $B_{0}=1.0 \mathrm{~T}$, the smallest beam diameter is about $4 \mu \mathrm{m}$ (Figure 4a). Focusing increases the energy spread (Figure 4b) and length (Figure 4c) of the pulse, besides distorting the pulse shape (Figure $4 d$ ). Note in Figure $4 b$ that the energy spread of the electron pulse increases markedly after focusing by the lens (at $z=10 \mathrm{~cm}$ ), due to the increased stochastic interaction (Boersch effect).

An electron pulse lengthens during propagation in free space (within the first $5 \mathrm{~cm}$ in Figure 4c) due to velocity spread of the electrons [38], but radio-frequency (RF) cavity electron sources can produce a chirped pulse (velocity higher at the back of the pulse) to counteract this effect, so that the electrons arrive at a specified plane longitudinally focused [19].

\section{Requirements for diffractive imaging}

Deriving an image of a particle through its diffraction pattern does not provide any information additional to what could be obtained from direct phase-contrast imaging with an ideal lens. However, diffractive imaging requires no focusing lenses after the specimen, thereby minimizing the loss of spatial resolution due to lens aberrations and energy broadening at beam crossovers, where the current density is high.

The RF cavity XFEL source, which produces pulses of duration $T \approx 100 \mathrm{fs}$, is also used for ultrafast electron diffraction (UED) experiments [22,23,39,40]. To illustrate the signal collection requirement associated with using such a source for diffractive imaging, consider a particle of diameter $d$ within a pulsed beam of diameter $D$, as in Figure 5 . The number of electrons diffracted by a single pulse is $N_{\mathrm{d}} \approx N_{\mathrm{p}}(d / D)^{2}\left(d / \lambda_{\mathrm{e}}\right)$, where $N_{\mathrm{p}}$ is the number of electrons per pulse and $\lambda_{\mathrm{e}}$ is the mean free path for elastic scattering. In what follows, we will take $N_{\mathrm{d}}=10^{3}$, since phases of the diffracted beams must be determined, although the value might be much lower for incoherent imaging and possibly a reconstruction algorithm could be used that does not require indexing a diffraction pattern from each particle [41]. Assuming $N_{\mathrm{p}} \approx 10^{6}, \lambda_{\mathrm{e}} \approx 1 \mu \mathrm{m}$ for $2.5-\mathrm{MeV}$ electrons, and $d \approx 10 \mathrm{~nm}$ (the size of a typical protein molecule), the required beam diameter is $D \approx\left(N_{\mathrm{p}} /\right.$ $\left.N_{\mathrm{d}}\right)^{1 / 2} d^{3 / 2} / \lambda_{\mathrm{e}}^{1 / 2} \approx 30 \mathrm{~nm}$. Comparison with Figure $4 \mathrm{a}$ shows that this diameter is below what is possible with 


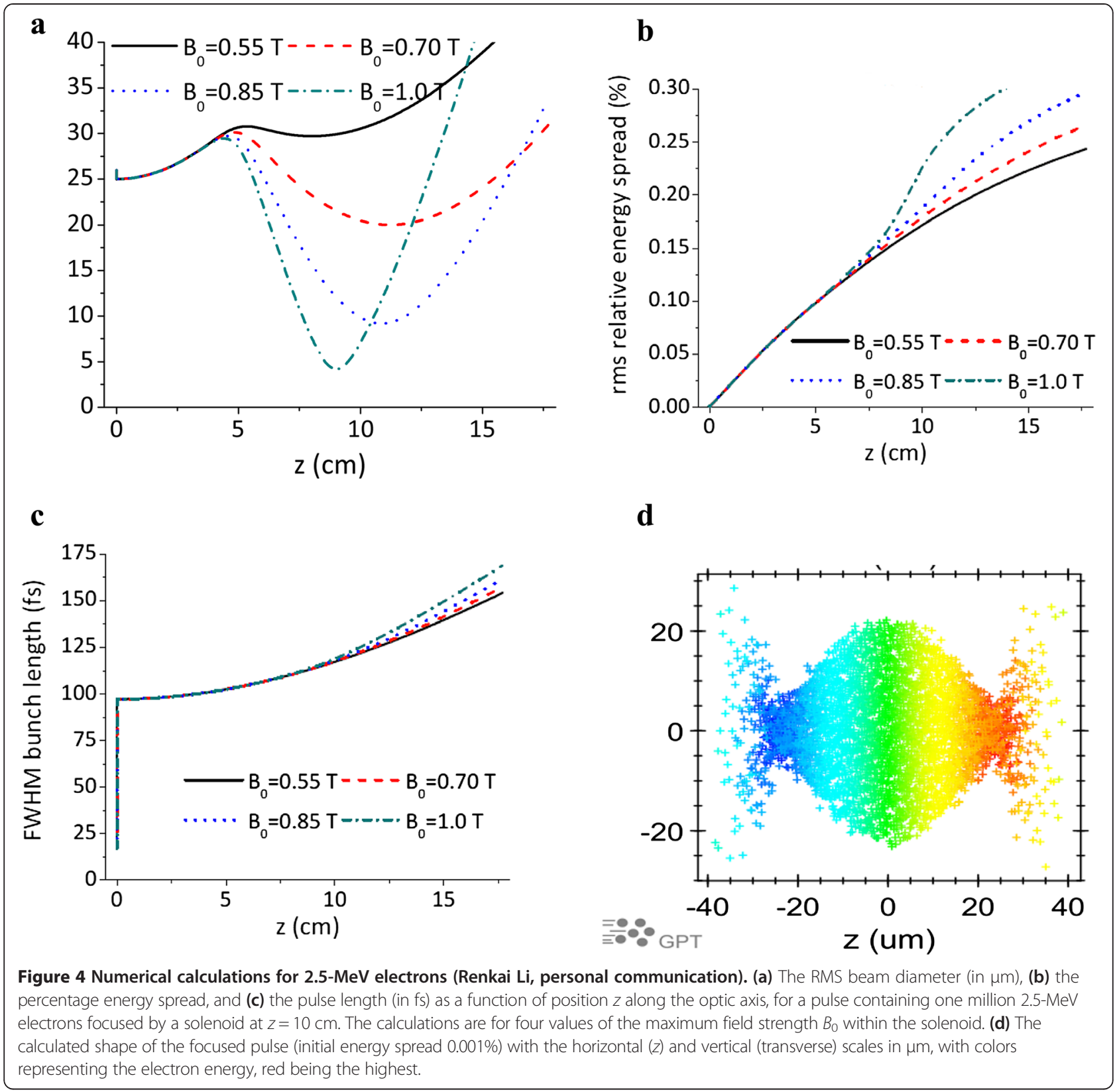

current instrumentation, although superconducting lenses or aberration-corrected optics would help. The associated current density is $J \approx\left(e N_{\mathrm{p}} / D^{2}\right) T^{-1}=1.6 \times 10^{12} \mathrm{~A} / \mathrm{cm}^{2}$, exceeding the estimated stochastic limit (Figure $3 \mathrm{~b}$ ) even when reduced (for 100-fs pulses) by a factor of 300 .

The above calculation assumes structure determination from single isolated molecules, which is the future goal of XFEL crystallography [9]. If instead the specimen consists of crystals of length $500 \mathrm{~nm}$ along $x_{-}, y_{-}$, and $z$-axes, the required beam diameter is $D \approx$ $\left(N_{\mathrm{p}} / N_{\mathrm{d}}\right)^{1 / 2} d^{3 / 2} / \lambda_{\mathrm{e}}^{1 / 2} \approx 11 \mu \mathrm{m}$, giving an instantaneous current density of $1.3 \mathrm{MA} / \mathrm{cm}^{2}$ at $2.5 \mathrm{MeV}$. Comparison with Figures $3 \mathrm{~b}$ and 4 shows that these requirements can be satisfied, so the quantity of the diffracted signal is sufficient.

However, we must also consider beam coherence, which determines the quality of the signal. For diffractive imaging with a beam of angular spread $\pm \alpha$, the transverse coherence length $\lambda_{\mathrm{T}}=\lambda /(2 \pi \alpha)$ must exceed the particle size or the unit cell size in the case of a crystal [10]. For a typical protein molecule, this implies $\lambda_{\mathrm{T}}>$ $10 \mathrm{~nm}$ and $\alpha<6.6 \mu \mathrm{rad}$ for $2.5-\mathrm{MeV}$ electrons $(\lambda=$ $0.42 \mathrm{pm})$ or $\alpha<60 \mu \mathrm{rad}$ for $100-\mathrm{keV}$ electrons $(\lambda=$ $3.7 \mathrm{pm})$. For XFEL $\mathrm{x}$-rays, whose wavelength $\lambda$ exceeds $100 \mathrm{pm}$, the beam divergence requirement is readily satisfied, but for electrons, this is not so. For example, 


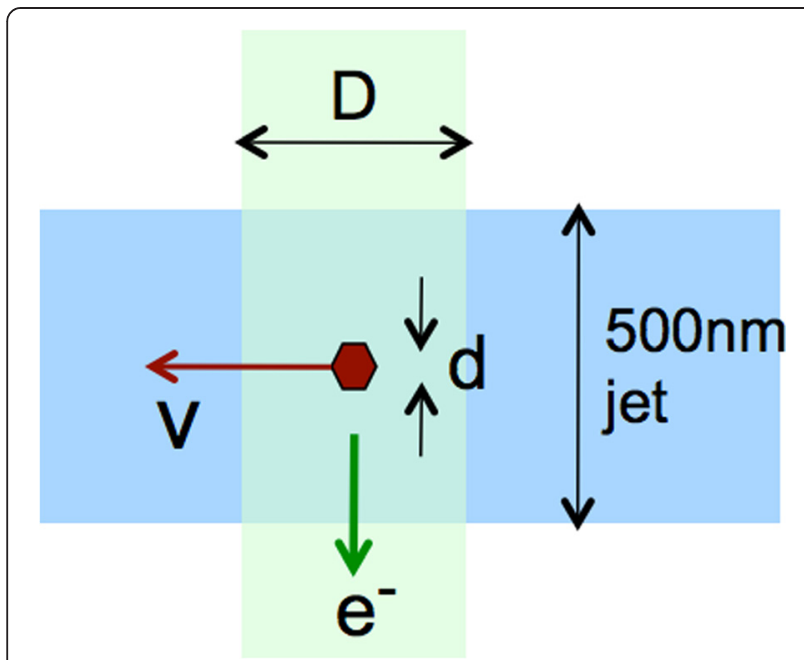

Figure 5 Particle/beam geometry for TEM serial

crystallography. Particles (diameter $d$ ) move at speed $v$ within a liquid jet (blue) and are irradiated by an electron beam (green) of diameter $\mathrm{D}$. The geometry for XFEL diffract-before-destroy is similar.

existing UED sources provide $\alpha \approx 300 \mu \mathrm{rad}$ and $\lambda_{\mathrm{T}}<$ $1 \mathrm{~nm}$ for a $2.5-\mathrm{MeV}$ beam diameter of $100 \mu \mathrm{m}$ [40] or $\lambda_{\mathrm{T}} \approx 3 \mathrm{~nm}$ for a $500-\mu \mathrm{m}$ spot at $100 \mathrm{keV}$ [39].

The angular divergence $\alpha$ is related to the beam brightness $B=J /\left(\pi \alpha^{2}\right)$, which cannot exceed the brightness of the electron source (unless pulse compression is used). For structural imaging of 10-nm molecular particles with 100 -fs pulses of $2.5-\mathrm{MeV}$ electrons, we need $\alpha<6.6$ $\mu \mathrm{rad}$ for adequate lateral coherence and $J=1.6 \times 10^{12} \mathrm{~A} /$ $\mathrm{cm}^{2}$ for sufficient signal $\left(N_{\mathrm{d}}=1,000\right)$, giving $B>10^{22} \mathrm{~A} /$ $\mathrm{cm}^{2} / \mathrm{sr}$. If the particles consist of 500-nm crystals, the requirements become $J=0.6 \mathrm{MA} / \mathrm{cm}^{2}$ and $B>4.4 \times 10^{15} \mathrm{~A} /$ $\mathrm{cm}^{2} / \mathrm{sr}$. As the brightness of existing $2.5-\mathrm{MeV}$ UED sources appears to be about $10^{10} \mathrm{~A} / \mathrm{cm}^{2} / \mathrm{sr}[22,40]$, source brightness is clearly a major problem.

For $100-\mathrm{keV}$ electrons, where $\lambda_{\mathrm{T}}>10 \mathrm{~nm}$ implies $\alpha<$ $60 \mu \mathrm{rad}$ and $N_{\mathrm{d}}=1,000$ requires $J=3.2 \times 10^{10} \mathrm{~A} / \mathrm{cm}^{2}$ for $N_{\mathrm{p}}=10^{6}$, the required brightness is $B=2.8 \times 10^{18} \mathrm{~A} /$ $\mathrm{cm}^{2} / \mathrm{sr}$. For $500-\mathrm{nm}$ crystals, this becomes $J=0.26 \mathrm{MA} /$ $\mathrm{cm}^{2}$ and $B=2.3 \times 10^{13} \mathrm{~A} / \mathrm{cm}^{2} / \mathrm{sr}$, although in this case, a crystal as thick as $500 \mathrm{~nm}$ would cause strong dynamical scattering [42] that would complicate interpretation of the diffraction pattern.

An electron source can be characterized by a brightness $B=I /(\pi \varepsilon)^{2}$, by a transverse emittance $\varepsilon=r \alpha$, where $r$ and $\alpha$ are the beam radius and divergence semi-angle, or by a normalized emittance: $\varepsilon^{*}=\gamma(v / c) \varepsilon$, where $v$ is the electron speed and $\gamma=\left(1-v^{2} / c^{2}\right)^{-1 / 2}$. Both $\varepsilon$ and $B$ are conserved in an ideal optical system but are degraded by Coulomb repulsion between electrons, which reduces $B$ and increases $\varepsilon$. An even more useful measure is the coherent fluence: $N_{\mathrm{c}}=N_{\mathrm{p}} \lambda^{2} / \varepsilon^{2}=\pi \lambda^{2} B$ T/e, which is unaffected by electron acceleration, beam focusing or apertures, space charge, or pulse compression but is degraded by lens aberrations and stochastic interaction [43]. For diffraction-contrast images, $N_{\mathrm{c}} \approx 1$ suffices, but for phase-contrast or diffractive imaging, $N_{\mathrm{c}}>10$ is required, whereas current UED systems have $N_{\mathrm{c}}<<1$ (Bryan Reed, personal communication), hence the need for electron gun development, which includes maximizing the extraction field at the photocathode, ensuring low capacitance (for short pulses), and reducing the thermal emittance by using a low cathode temperature.

Cold-atom sources promise a substantial improvement in coherence and control over Coulomb repulsion forces $[44,45]$. Electrons are emitted over a relatively large area by near-threshold laser ionization of an ultracold gas (e.g., $\mathrm{Rb}$ atoms). By adjusting the shape of the laser field, the shape of the emitted electron bunch can be tailored to ensure low beam divergence and allow standard electron optics to reverse the Coulomb expansion of each bunch [46,39]. For 5-ns $1-\mathrm{keV}$ bunches containing $10^{5}$ electrons, a transverse coherence length of $10 \mathrm{~nm}$ was achieved and a brightness exceeding $10^{11}$ $\mathrm{A} / \mathrm{cm}^{2} / \mathrm{sr}$ [46], corresponding to $N_{\mathrm{c}}>1.5 \times 10^{-3}$. More recently, 150-ps bunches of $1-\mathrm{keV}$ electrons have been produced with a coherence length $\approx 4 \mathrm{~nm}[47,48]$. With further development, it seems that pulsed electron beams could perform serial crystallography of nanocrystals, with structure determination from isolated macromolecules a more remote possibility.

\section{Diffract without destroy}

The very large $x$-ray fluence produced by a free-electron laser $\left(\approx 10^{12}\right.$ photons per pulse) results in a dose rate of the order of $10^{21} \mathrm{~Gy} / \mathrm{s}$ [49], and a protein molecule is destroyed within $50 \mathrm{fs}$ [50]. From Equation 1, the electron dose rate is $\mathrm{d} D(\mathrm{~Gy}) / \mathrm{d} t=(\mathrm{J} / \rho)\left[E_{\mathrm{m}}(\mathrm{eV}) / \lambda_{\mathrm{i}}\right]$, where $E_{\mathrm{m}}$ $(\mathrm{eV})$ is the mean energy loss in $\mathrm{eV}$ per inelastic collision. For $J=10^{8} \mathrm{~A} / \mathrm{cm}^{2}$ (100-fs pulses containing $10^{6}$ electrons, focused to $1 \mu \mathrm{m})$, the dose rate is below $10^{14} \mathrm{~Gy} /$ $\mathrm{s}$, seven orders of magnitude less than the XFEL case.

If we characterize electron damage by a critical fluence $D_{\text {c }}$ measured at a low dose rate, destruction within a time $T$ requires $J T>D_{\mathrm{c}}$. For $T=100 \mathrm{fs}$ and $D_{\mathrm{c}}=0.01 \mathrm{C} /$ $\mathrm{cm}^{2}$ (typical of a sensitive organic material) [42], the current density would need to exceed $10^{11} \mathrm{~A} / \mathrm{cm}^{2}$, higher than what is achievable or required for $0.3-\mathrm{nm}$ imaging from 500-nm crystals. So reliance on nuclear inertia to outrun radiation damage is not necessary with electrons, thanks to their higher diffracted signal in relation to the energy deposition.

Only if $J>10^{12} \mathrm{~A} / \mathrm{cm}^{2}$ were possible (the value that might be needed for imaging single-molecule particles) would the molecules be destroyed by a single 100-fs pulse. 


\section{Using longer pulses for electrons}

So far, we have assumed 100-fs pulses because such pulses are appropriate for XFEL measurements [50] and because the corresponding electron pulses are available for UED experiments [22,23]. However, King et al. [51] have reported hydrodynamic simulations that predict an image resolution of $0.4 \mathrm{~nm}$ within a $10-\mathrm{nm}$ molecule, for 2-ps pulses containing ten million $100-\mathrm{keV}$ electrons focused to 100 -nm diameter. The ability to use longer (ps or ns) pulses would make electrons more feasible for serial crystallography. For a given signal per pulse, the current density would be reduced, easing the Coulomb repulsion problem and the source brightness requirement.

Electrons have relatively large elastic scattering cross sections: $2 \times 10^{-23} \mathrm{~m}^{2}$ at $2.5 \mathrm{MeV}$ or $7 \times 10^{-23} \mathrm{~m}^{2}$ at $100 \mathrm{keV}$, compared to $\approx 10^{-27} \mathrm{~m}^{2}$ per carbon atom for 8 $\mathrm{keV}$ x-rays [26]. Therefore, 100-fs pulses containing ten million electrons should produce almost as large a diffraction signal as 8 -keV XFEL pulses containing $10^{12}$ photons, for a nanocrystal of the same size. These electron pulses would therefore be capable of providing 0.4$\mathrm{nm}$ resolution from biomolecules, as in previous XFEL measurements. A pulse duration of 2 ps rather than $100 \mathrm{fs}$ does not change the calculation, so the prediction of King et al. [51] appears reasonable in terms of spatial resolution. However, focusing a $100-\mathrm{keV}$ beam (with $10^{7}$ electrons per pulse) down to 100-nm diameter implies a current of $0.8 \mathrm{~A}$ and current density of $8,000 \mathrm{MA} / \mathrm{cm}^{2}$, which appears unattainable (see Figure 3a).

Using a $2-\mu \mathrm{m}$-diameter beam, as in many XFEL measurements, would reduce the current density to $20 \mathrm{MA} /$ $\mathrm{cm}^{2}$, well above the stochastic limit $\left(\approx 2,000 \mathrm{~A} / \mathrm{cm}^{2}\right)$ for a continuous beam. But if that limit is much lower for 2ps pulses and if space-charge broadening can be reversed by lens focusing [39], the fluence associated with each 2 ps pulse would be $4 \times 10^{-5} \mathrm{C} / \mathrm{cm}^{2}$, well below the characteristic dose $\left(\approx 10^{-2} \mathrm{C} / \mathrm{cm}^{2}\right)$ for biological material exposed to $100-\mathrm{keV}$ electrons [42]. For a beam divergence below $60 \mu \mathrm{rad}$, however, a source brightness $B>2.5 \times$ $10^{10} \mathrm{~A} / \mathrm{cm}^{2} / \mathrm{sr}$ is required.

It has been suggested that the damage that degrades the diffracted signal occurs more slowly with electrons than with $\mathrm{x}$-rays, electrons producing less nuclear displacement for the same energy deposition. However, both electrons and $\mathrm{x}$-rays deliver energy to the electrons of the sample and the mechanisms of energy transfer to nuclei are presumably the same and take place within 1 fs [52]. At high particle flux, atomic motion is limited by the inertia of the nuclei but at lower flux by the energy-deposition rate. It seems unlikely that electron sources will attain the brightness of an XFEL source, which allows diffraction measurements to take advantage of the nuclear inertia, but the aim of serial crystallography is to obtain diffraction data, not to outrun damage.
Another possibility is that the diffracted signal of electrons persists longer than with $\mathrm{x}$-rays, for the same energy deposition. This idea is related to the fact that electrons diffract from the electrostatic field of atomic nuclei, whereas $\mathrm{x}$-rays diffract from the surrounding electrons. If atoms of the specimen became totally ionized, with all electrons stripped from the nuclei, the $\mathrm{x}$ ray diffraction signal would disappear but electrons would still be diffracted. However, this situation is believed to occur only for very high $\mathrm{x}$-ray intensity $\left(>10^{13}\right.$ photons/pulse, with a 100-nm focus). Through photoabsorption and Auger emission, a typical XFEL pulse strips about three electrons from each carbon atom, with only a modest change to the $\mathrm{x}$-ray diffraction pattern. This change is represented by an electronic component $R_{\text {elec }}$ in the $R$-factor that describes the reduction in data quality, and appears faster than the degradation $R_{\text {nucl }}$ due to the displacement of atomic nuclei. For $12-\mathrm{keV} \mathrm{x}$-rays and below $10^{12}$ photons per pulse, $R_{\text {elec }}<15 \%$ (acceptable damage) up to $40 \mathrm{fs}$, after which $R_{\text {nucl }}$ becomes important [15]. So even if the diffraction of electrons were sensitive to $R_{\text {nucl }}$ only, this is unlikely to be a substantial advantage.

\section{Continuous-beam serial crystallography}

The limiting case of a long pulse is a continuous beam, as employed in most electron microscopes. Deponte et al. tested their liquid jet injector in a differentially pumped sample chamber of a 200-kV TEM and photographed the jet under different flow conditions [24]. For a jet velocity $v \approx 100 \mathrm{~m} / \mathrm{s}$, jet diameters down to $350 \mathrm{~nm}$ were found to be feasible. However, gold particles within a water jet (mass fraction $\approx 50 \mathrm{ppm}$ ) failed to produce observable diffraction spots.

For the case of $10-\mathrm{nm}$ protein molecules, we can analyze the situation for a $200-\mathrm{kV}$ cold field-emission source with brightness $B=6 \times 10^{9} \mathrm{~A} / \mathrm{cm}^{2} / \mathrm{sr}$. Assuming a beam current $I=10 \mathrm{nA}$, a beam of diameter $D=(I / B)^{1 / 2}$ $\left(\pi \alpha^{2}\right)^{-1 / 2}=120 \mathrm{~nm}$ would provide low enough beam divergence $(\alpha=60 \mu \mathrm{rad})$ for diffractive imaging, with a current density $J=68 \mathrm{~A} / \mathrm{cm}^{2}$. For a jet velocity $v=100 \mathrm{~m} / \mathrm{s}$, the transit time of a particle within the beam is $T=D / \nu \approx$ $1.2 \mathrm{~ns}$ and the electron fluence is $J T=8.2 \times 10^{-8} \mathrm{C} / \mathrm{cm}^{2}$, far below the damage dose $\approx 10^{-2} \mathrm{C} / \mathrm{cm}^{2}$. The number of electrons intercepted by each molecular particle during transit is $N_{\mathrm{i}} \approx(J T / e)(10 \mathrm{~nm})^{2}=0.5$, and (for elastic mean free path $\lambda_{\mathrm{e}} \approx 300 \mathrm{~nm}$ ) the number diffracted is $N_{\mathrm{d}} \approx N_{\mathrm{i}}$ $\left(10 \mathrm{~nm} / \lambda_{\mathrm{e}}\right)=0.017$, obviously too low. For an equiaxed crystal of diameter $d=100 \mathrm{~nm}$, the number of diffracted electrons per pulse would be $N_{\mathrm{d}} \approx(J T / e)\left(d^{3} / \lambda_{\mathrm{e}}\right) \approx 17$. Besides the diffraction signal being too weak, there would also be a large inelastic background to the diffraction pattern, although most that could be removed by energy filtering. 
Another problem is molecular tumbling, typically $10^{8} \mathrm{rad} / \mathrm{s}$ for a macromolecule. Within a transit time of $380 \mathrm{~s}$, the molecule would rotate by $38 \mathrm{mrad}$ and produce a blurred diffraction pattern. Laser alignment is a possibility [53,54] and has recently been achieved [55]. With nanocrystalline specimens, the tumbling rate could be much less and stabilization might be unnecessary.

Particle tumbling is avoided altogether in singleparticle imaging of molecules on a thin substrate [56] where the use of cross-correlation (to determine relative orientation) allows a reduction in the radiation dose relative to visual inspection [57]. The orientation issue is also avoided by the use of crystalline arrays of molecules in the same orientation [58]. Phase-contrast bright-field TEM imaging is normally used for these studies; annular dark-field STEM imaging has been explored [59] but judged to offer a worse signal/noise ratio [60].

\section{Conclusions}

Serial crystallography using an XFEL was developed as a way of determining the molecular structure of biostructures (such as proteins) injected into the beam in a liquid jet or aerosol. The XFEL pulses are less than $100 \mathrm{fs}$ in width but contain $\approx 10^{12}$ photons, so a diffraction pattern is obtained within the first $40 \mathrm{fs}$ of each pulse, before atoms of the specimen have time to move, making radiation damage no longer a limiting factor. By combining diffraction information from many pulses, threedimensional molecular structures have been determined with 0.2- to 0.4-nm resolution from small crystals. With anticipated engineering advances, the same resolution may eventually be possible from isolated molecules [9].

Employing short bunches of electrons for the same purpose suffers from two major problems: Coulomb repulsion between the electrons in each bunch and the beam coherence requirement for phase-contrast imaging. Space-charge and stochastic repulsion, together with the associated energy broadening, make lens focusing problematic. The use of diffractive imaging avoids the need for post-specimen focusing, but Coulomb repulsion between the incident electrons (before they arrive at the sample) limits the current density, the signal from each molecule, and the spatial resolution of the analysis. Both space-charge and stochastic repulsion are reduced by using $\mathrm{MeV}$ rather than $\mathrm{keV}$ electron energies, but the repulsion problem still appears severe.

A second problem is the relatively low lateral coherency of an electron beam, arising from the shorter wavelength of the electrons compared to $\mathrm{x}$-rays and the lower brightness of electron sources relative to the XFEL. Cold-atom sources offer the possibility of a substantial improvement in coherence, together with some control over the Coulomb repulsion effect.
Using longer electron pulses would reduce the current density and coherency requirements. Even so, substantial further development of higher-brightness electron sources, preferably with the use of $\mathrm{MeV}$ energies, appears necessary for three-dimensional diffractive imaging of beam-sensitive specimens with close to atomic resolution and subnanosecond time resolution.

Serial crystallography is also possible by particle injection into the continuous electron beam of a TEM. However, the limited brightness of field-emission sources is a major obstacle, together with molecular tumbling (unless laser alignment is used). The techniques of cryo-TEM and single-particle TEM imaging avoid these problems by measuring a large number of stationary molecules, directly imaged by electron lenses, and have benefited from the recent availability of fast-readout direct-recording detectors. The molecules are then not in their natural wet state but the need for large crystals is avoided, making these TEM techniques competitive with $\mathrm{x}$-ray crystallography.

In conclusion, short $(<100 \mathrm{fs})$ pulses of electrons will not replicate the XFEL experience of outrunning primary ionization damage, although secondary and tertiary damage is easily overcome because of the longer timescales [61]. But thanks to the higher diffractive power of electrons, it is not necessary to outrun all damage in order to obtain a signal sufficient to determine the three-dimensional structure of macromolecules at near-atomic resolution, at least using nanocrystalline specimens, and given sufficient further development of high-brightness electron sources.

During this time, XFEL techniques will be moving from crystals towards single molecules and many important structures will have been solved using XFEL, single-particle imaging, or cryomicroscopy in the TEM. Even so, pulsed electron beams are attractive because of their ability to provide time resolution in both repetitive (stroboscopic) and non-repetitive (single-shot) modes, allowing such things as 'molecular movies' and the study of phase transitions [43,51]. Incoherent contrast will suffice for many of these applications, and single-pulse dark-field images with subnanometer resolution may be possible with an RF gun source and aberration-corrected lenses [43].

Competing interests

The author declares that he has no competing interests.

\section{Acknowledgements}

The author thanks Nigel Browning, Bob Glaeser, Stefan Hau-Riege, Renkai Li, Bryan Reed, John Spence, and Yimei Zhu for the helpful discussions and the Natural Sciences and Engineering Research Council of Canada for the financial support.

Received: 24 July 2014 Accepted: 18 November 2014

Published online: 25 March 2015

\footnotetext{
References

1. Spence, JCH: Diffractive (lensless) imaging. In: Hawkes, PW, Spence, JCH (eds.) Science of Microscopy, vol. 2, pp. 1196-1227. Springer, New York (2007)
} 
2. Glaeser, RM: Electron crystallography: present excitement, a nod to the past, anticipating the future. J Struct Biol 128, 3-14 (1999)

3. Cudney, B: Protein crystallization and dumb luck. The Rigaku Journal 16, 1-7 (1999). http://www.rigaku.com/downloads/journal/Nol16.2.1999/ cudney.pdf

4. Adrian, M, Dubochet, J, Lepault, J, McDowall, AW: Cryo-electron microscopy of viruses. Nature 308, 32-36 (1984). doi:10.1038/308032a0

5. McMullan, G, Faruqi, AR, Clare, D, Henderson, R: Comparison of optimal performance at $300 \mathrm{kV}$ of three direct electron detectors for use in low dose electron microscopy. Ultramicroscopy 147, 156-163 (2014)

6. Li, X, Mooney, P, Zheng, S, Booth, CR, Braunfeld, MB, Gubbens, S, Agard, DA, Cheng, $Y$ : Electron counting and beam-induced motion correction enable near-atomic-resolution single-particle cryo-EM. Nat Methods 10, 584-590 (2013)

7. Glaeser, RM: Methods for imaging weak-phase objects in electron microscopy. Rev. Sci. Instrum. 84, 111101 (1013). doi:10.1063/1.4830355

8. Frank, J: Three-dimensional electron microscopy of macromolecular assemblies: visualization of biological molecules in their native state. Oxford University Press, Oxford (2006). ISBN 978-0-19-518218-7

9. Spence, J, Weierstall, U, Chapman, H: X-ray lasers for structural and dynamic biology. Rep Prog Phys 75, 102601 (2012)

10. Spence, JCH, Weierstall, U, Howells, M: Coherence and sampling requirements for diffractive imaging. Ultramicroscopy 101, 149-152 (2004)

11. McEwen, BF, Downing, KH, Glaeser, RM: The relevance of dose-fractionation in tomography of radiation-sensitive specimens. Ultramicroscopy 60, 357-373 (1995)

12. Chapman, $\mathrm{H}$, et al.: Femtosecond X-ray protein nanocrystallography. Nature 460, 73-77 (2011). doi:10.1038/nature09750

13. Frank, $M$, et al.: Femtosecond X-ray diffraction from two-dimensional protein crystals. IUCrJ 1, 95-100 (2014). doi:10.1107/S2052252514001444

14. Seibert, $M$, et al.: Single mimivirus particles imaged on-the-fly with an X-ray laser. Nature 470, 782011 (2011)

15. Neutze, R, Wout, R, van der Spoel, D, Weckert, E, Hajdu, J: Potential for biomolecular imaging with femtosecond $\mathrm{X}$-ray pulses. Nature 406, 752-757 (2000)

16. Caleman, C, Bergh, M, Scott, HA, Spence, JCH, Chapman, H, Timneanu, N: Simulations of radiation damage in biomolecular nanocrystals by femtosecond X-ray pulses. J Modern Optics 58, 1486-1497 (2011)

17. Hau-Riege, SP, London, RA, Chapman, HN: Soft-x-ray free-electron-laser interaction with materials. Phys Rev E 98, 046403 (2007)

18. Kern, J, Roberto Alonso-Mori, J, Tran, R, Hattne, J, Gildea, RJ, Echols, N, Glöckner, C, Hellmich, J, Laksmono, H, Sierra, RG, Lassalle-Kaiser, B, Koroidov, S, Lampe, A, Han, G, Gul, S, DiFiore, D, Milathianaki, D, Fry, AR, Miahnahri, A, Schafer, DW, Messerschmidt, M, Seibert, MM, Koglin, JE, Sokaras, D, Weng, T-C, Sellberg, J, Latimer, MJ, Grosse-Kunstleve, RW, Zwart, PH, White, WE, Glatzel, P, Adams, PD, Bogan, MJ, Williams, GJ, Boutet, S, Messinger, J, Zouni, A, Sauter, NK, Yachandra, VK, Bergmann, U, Yano, J: Simultaneous femtosecond $X$-ray spectroscopy and diffraction of photosystem $\|$ at room temperature. Science 340, 491-495 (2013)

19. van Oudheusden, T, Pasmans, PLEM, van der Geer, SB, de Loos, MJ, van der Wiel, MJ, Luiten, OJ: Compression of subrelativistic space-charge-dominated electron bunches for single-shot femtosecond electron diffraction. Phys Rev Lett 105, 264801 (2010)

20. Chatelain, RP, Morrison, VR, Godbout, C, Siwick, BJ: Ultrafast electron diffraction with radio-frequency compressed electron pulses. Appl Phys Lett 101, 081901 (2012)

21. Gao, M, Jean-Ruel, H, Cooney, RR, Stampe, J, de Jong, M, Harb, M, Scianini, G, Moriena, G, Miller, RJD: Full characterization of RF compressed femtosecond electron pulses using ponderomotive scattering. Opt Express 20, 12048-12058 (2012)

22. Hastings, JB, Rudakov, FM, Dowell, DH, Schmerge, JF, Cardoza, JD, Castro, JM, Gierman, SM, Loos, H, Weber, PM: Ultrafast time-resolved electron diffraction with megavolt electron beams. Appl Phys Lett 89, 184109 (2006)

23. Zhu, P, Cao, J, Zhu, Y, Geck, J, Hidaka, Y, Pjerov, S, Ritschel, T, Berger, H, Shen, $Y$, Tobey, R, Hill, JP, Wang, XJ: Dynamic separation of electron excitation and lattice heating during the photoinduced melting of the periodic lattice distortion in 2H-TaSe 2 . Appl Phys Lett 103, 071914 (2013)

24. Deponte, DP, Mckeown, JT, Weierstall, U, Doak, RB, Spence, JCH: Towards ETEM serial crystallography: electron diffraction from liquid jets. Ultramicroscopy 111, 824-827 (2011)
25. Garrett, BC, et al.: The role of water in electron-initiated processes and radical chemistry: issues and scientific advances. Chem Rev 105, 355-389 (2005)

26. Henderson, R: The potential and limitations of neutrons, electrons and X-rays for atomic resolution microscopy of unstained biological molecules. Quarterly Reviews of Biophys 2, 171-193 (1995)

27. Egerton, RF: Mechanisms of radiation damage in beam-sensitive specimens, for TEM accelerating voltages between 10 and $300 \mathrm{kV}$. Microsc Res Tech 75, 1550-1556 (2012). doi:10.1002/jemt.22099

28. Spence, JCH, Howells, MR: Synchrotron soft X-ray and field-emission electron sources: a comparison. Ultramicroscopy 93, 213-222 (2002)

29. Egerton, RF: Choice of operating voltage for a transmission electron microscope. Ultramicroscopy 145, 85-93 (2014). doi:1016/j.ultramic.2013.10.019

30. Howells, MR, Beetz, T, Chapman, HN, Cui, C, Holton, JM, Jacobsen, CJ, Kirz, J, Lima, E, Marchesini, S, Miao, SH, Sayre, D, Shapiro, DA, Spence, JCH, Starodub, $\mathrm{D}$ : An assessment of the resolution limitation due to radiation-damage in $\mathrm{X}$-ray diffraction microscopy. J Elec Spectr Rel Phenom 170, 4-12 (2009)

31. Shen, Q, Bazarov, I, Thibault, P: Diffractive imaging of nonperiodic materials with future coherent X-ray sources. J Synchrotron Radiation 11, 432-438 (2004). doi:10.1107/S0909049504016772

32. King, WE, Campbell, GH, Frank, A, Reed, B, Schmerge, JF, Siwick, BJ, Stuart, BC, Weber, PM: Ultrafast electron microscopy in materials science, biology, and chemistry. J Appl Phys 97, 1-27 (2005)

33. Kruit, $\mathrm{P}$, Jansen, GH: Space charge and statistical Coulomb effects. In: Orloff, J (ed.) Handbook of Charged Particle Optics, pp. 275-318. Springer, New York (1997)

34. Spehr, R: Broadening of charged particle microprobes by stochastic Coulomb interactions. Optik 70, 109-114 (1985)

35. Berger, A, Spehr, R, Rose, $\mathrm{H}$ : Computer simulations of trajectory displacements with consideration of the energy width of the beam. Optik 86, 77-85 (1990)

36. Li, R, Huang, W, Du, Y, Yan, L, Du, Q, Shi, J, Hua, J, Chen, H, Du, T, Xu, H, Tang, C: Single-shot continuously time-resolved MeV ultrafast electron diffraction. Rev Sci Instrum 81, 036110 (2010)

37. Armstrong, MR, Reed, BW, Torralva, BR, Browning, ND: Prospects for electron imaging with ultrafast time resolution. Appl Phys Lett 90, 114101 (2007)

38. Siwick, BJ, Dwyer, JR, Jordan, RE, Miller, RJD: Ultrafast electron optics: propagation dynamics of femtosecond electron packets. J Appl Phys 92, 1643-1648 (2002)

39. van Oudheusden, T, de Jong, EF, van der Geer, SB, Op't Root, WPEM, Luiten, OJ: Electron source concept for single-shot sub-100 fs electron diffraction in the $100 \mathrm{keV}$ range. J Appl Phys 102, 093501 (2007)

40. Wang, XJ, Xiang, D, Kim, TK, Ihee, H: Potential of femtosecond electron diffraction using near-relativistic electrons from a photocathode rf electron gun. J Korean Phys Soc 48, 390-396 (2006)

41. Saldin, DK, Schneerson, VL, Fung, R, Ourmazd, A: Structure of isolated biomolecules obtained from ultrashort $x$-ray pulses: exploiting the symmetry of random orientations. J Phys C 21, 134014 (2009)

42. Reimer, L, Kohl, H: Transmission Electron Microscopy, 5th edn. Springer, New York (2008)

43. Reed, BW, Armstrong, MR, Browning, ND, Campbell, GH, Evans, JE, LaGrange, T, Masiel, DJ: The evolution of ultrafast electron microscope instrumentation. Microsc Microanal 15, 272-281 (2009). doi:10.1017/\$1431927609090394

44. Van der Geer, SB, de Loos, MJ, Vredenbregt, EJD, Luiten, OJ: Ultracold electron source for single-shot, ultrafast electron diffraction. Microsc Microanal 16, 282-289 (2009). doi:10.1017/S143192760909076X

45. Vredenbregt, E, Luiten, J: Cool beams in great shape. Nat Phys 7, 747-748 (2011)

46. McCulloch, AJ, Sheludko, DV, Saliba, SD, Bell, SC, Junker, M, Nugent, KA, Scholten, RE: Arbitrarily shaped high-coherence electron bunches from cold atoms. Nat Phys 7, 785-788 (2011)

47. McCulloch, AJ, Sheludko, DV, Junker, M, Scholten, RE: High-coherence picosecond electron bunches from cold atoms. Nat Commun 4, 1692 (2013). doi:10.1038/ncomms2699

48. Engelen, WJ, van der Heijden, MA, Bakker, DJ, Vredenbregt, EJD, Luiten, OJ: High-coherence electron bunches produced by femtosecond photoionization. Nat Commun 4, 1693 (2013). doi:10.1038/ncomms2700

49. Boutet, $\mathrm{S}$, et al.: High-resolution protein structure determination by serial femtosecond crystallography. Science 335, 362-364 (2012)

50. Barty, A, et al.: Self-terminating diffraction gates femtosecond X-ray nanocrystallography measurements. Nature Photonics 6, 35-40 (2011)

51. King, WP, Armstrong, MR, Bostanjoglo, O, Reed, B: High-speed electron microscopy. In: Hawkes, PW, Spence, JCH (eds.) Science of Microscopy, p. 430. Springer, New York (2007) 
52. Spence, JCH, Vecchione, T, Weierstall, U: A coherent photofield source for fast diffractive and point-projection imaging. Phil Mag 90, 14-28 (2010)

53. Stapelfeldt, $H$, Seideman, T: Aligning molecules with strong laser pulses. Rev Mod Phys 75, 543-557 (2003)

54. Spence, JCH, Schmidt, K, Wu, J, Hembree, G, Weierstall, U, Doak, B, Fromme, $P$ : Diffraction and imaging from a beam of laser-aligned proteins: resolution limits. Acta Cryst A 61, 237-245 (2005)

55. Küpper, J, et al.: X-ray diffraction from isolated and strongly aligned gas-phase molecules with a free-electron laser. Phys Rev Lett 112, 083002 (2014)

56. Frank, J: Single-particle reconstruction of biological macromolecules in electron microscopy - 30 years. Quart Rev Biophys 42, 139-158 (2009)

57. Saxton, WO, Frank, J: Motif detection in quantum noise-limited electron micrographs by cross-correlation. Ultramicroscopy 2, 219-227 (1977)

58. Henderson, R, Unwin, PNT: Three-dimensional model of purple membrane obtained by electron microscopy. Nature 257, 28-31 (1975)

59. Andrews, DW, Yu, AHC, Ottensmeyer, FP: Automatic selection of molecular images from dark field electron micrographs. Ultramicroscopy 19, 1-14 (1986)

60. Rez, P: Comparison of phase contrast transmission electron microscopy with optimized scanning transmission annular dark-field imaging for protein imaging. Ultramicroscopy 96, 117-124 (2003)

61. Warkentin, M, Badeau, R, Hopkins, JB, Mulichak, AM, Keefe, LJ, Thorne, RE: Global radiation damage at 300 and $260 \mathrm{~K}$ with dose rates approaching 1MGy s ${ }^{-1}$. Acta Cryst D 68, 124-133 (2012). doi:10.1107/S0907444911052085

\section{Submit your manuscript to a SpringerOpen ${ }^{\circ}$ journal and benefit from:}

- Convenient online submission

- Rigorous peer review

- Immediate publication on acceptance

- Open access: articles freely available online

- High visibility within the field

- Retaining the copyright to your article

Submit your next manuscript at $\gg$ springeropen.com 